\title{
Phase behavior of a mixture of platelike colloids and nonadsorbing polymer
}

\author{
F. M. van der Kooij, M. Vogel, and H. N. W. Lekkerkerker \\ Van't Hoff Laboratory for Physical and Colloid Chemistry, Debye Institute, Utrecht University, Padualaan 8 , \\ 3584 CH Utrecht, The Netherlands
}

(Received 24 May 2000)

\begin{abstract}
Suspensions of sterically stabilized colloidal gibbsite platelets have recently been found to exhibit both an isotropic-nematic $(I-N)$ and a nematic-columnar $(N-C)$ phase transition. In the present paper we show that depletion attraction, brought about by the addition of nonadsorbing polymer, enriches the phase behavior of these platelet suspensions even further. Pronounced broadening of the isotropic-nematic gap occurs, with purely nematic samples re-entering the biphasic state by the addition of nonadsorbing polymer. At the same time, depletion attraction enhances size fractionation between coexisting phases, which actually provides an effective means for reducing the polydispersity of the suspensions. An additional isotropic phase emerges, which leads to the appearance of several three-phase equilibria and even a four-phase equilibrium. We explain the observed topology of the phase diagram by the interplay between depletion attraction and the platelets' polydispersity.
\end{abstract}

PACS number(s): 82.70.Dd, 64.70.Md

\section{INTRODUCTION}

The addition of nonadsorbing polymer to a suspension of colloidal particles gives rise to a so-called depletion attraction, which originates from the overlap of the depletion zones (from which the polymer expelled) as the colloids approach. For separations between the particle surfaces smaller than the size of the polymer coil, the osmotic pressure of the polymer $\Pi_{p}$ gives rise to a depletion attraction between the colloids:

$$
W_{\mathrm{dep}} \simeq-\Pi_{p} V_{\mathrm{ov}},
$$

where $V_{\mathrm{ov}}$ is the volume by which the depletion zones of the colloids overlap. The theoretical framework for the depletion phenomenon was first presented by Asakura and Oosawa [1] in 1954, and put forward independently and extended by Vrij [2] in 1976. Classic examples of depletion attraction are the creaming of latex droplets in the presence of added polymer [3], and the aggregation of red blood cells (rouleaux formation) under the influence of proteins in the blood serum [4]. In particular, the application of depletion attraction to induce phase separation has raised a substantial interest in experiments $[5,6]$, theory $[7,8]$, and simulations $[9,10]$ over the last decades (for a review, see Ref. [11]). The colloid-polymer model that is almost exclusively studied in these model studies is that of hard-sphere colloids and polymers. The phase diagram of such mixtures has been shown to be determined by the range and the depth of the depletion attraction, which in the experiment can be tuned by the size and concentration of the polymer. A prominent feature of the sphere-polymer phase behavior is the appearance of a (dilute) colloidal gas $(G)$ and a (more concentrated) colloidal liquid $(L)$ phase, taking the place of a fluid phase, in cases where the polymer size exceeds one-third of the size of the sphere $[12,13]$. By conjunction of the subsequent $G-L, G-S$, and $L-S$ two-phase coexistence regions [where $S$ denotes the solid (colloidal crystal) phase], the depletion-induced attraction ultimately results in the emergence of a gas-liquid-solid $(G-L-S)$ triple coexistence region.

Compared to the phase behavior of spherical colloids and polymer, the phase behavior of mixtures of anisometric (i.e., rod- or platelike) particles and polymer is even richer due to the possibility of forming liquid-crystal phases [14]. Already in the 1940's, this phenomenon was exploited as an effective means for virus purification [15]. The underlying principle is that depletion attraction induces phase separation at lowvirus concentrations, yielding a highly concentrated liquidcrystal phase in coexistence with a very dilute isotropic phase. A more comprehensive understanding of the rodpolymer phase diagram has been raised by experiments on well-defined colloidal model systems of rods and polymers [16-18], theory [19], and simulations [20]. Next to broadening of the isotropic-nematic coexistence region, depletion attraction is found to enrich the phase behavior of rods by enhancing the stability of liquid-crystal phases (nematic, smectic) and by introducing an additional isotropic or nematic phase. The actual effect of the attraction depends on the range and depth of the depletion potential, as well as on the anisometry and polydispersity of the rods $[19,20]$.

Largely unexplored, however, is the phase behavior of mixtures of platelike colloids and polymer. While the phenomenon of depletion attraction does play a role in drilling fluids comprising platelike clay particles and polymer, the attraction thus raised, causes aggregation and gelation rather than equilibrium phase transitions [21]. This behavior may actually point at an intrinsic tendency of plate-polymer mixtures to form nonequilibrium states, originating from the relatively large overlap volume of the depletion zones of (parallel) plates compared to that of rods or spheres. Due to this geometrical difference, one expects that the depletion attraction in the case of plates is particularly fierce. Furthermore, from an experimental point of view, clay suspensions are not an obvious model system to study depletion-induced phase transitions, as they exhibit gelation even in the absence of a depletion attraction [22,23]. 


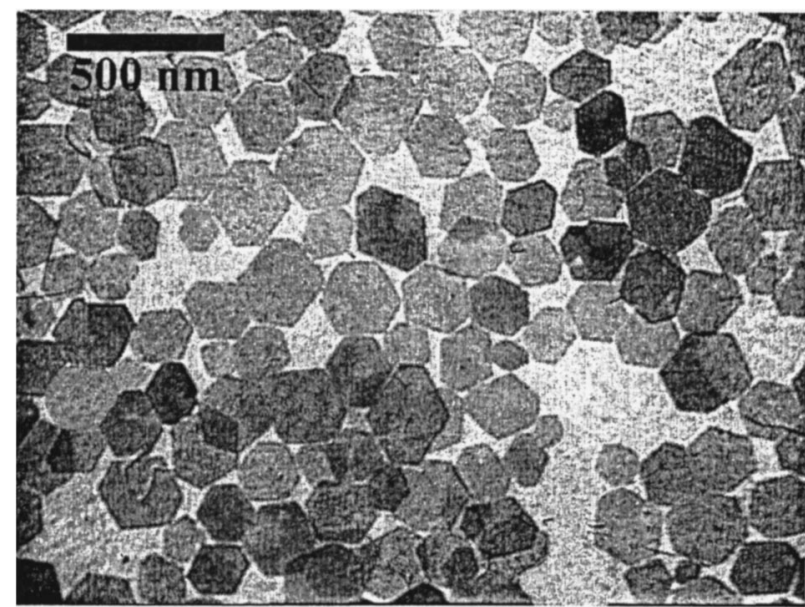

FIG. 1. TEM micrograph of the colloidal platelets used in this paper.

The objective of the present paper is to explore the phase diagram of a well-defined model system of colloidal platelets and nonadsorbing polymer. The adopted suspension of sterically stabilized platelets is unique in the sense that it exhibits both an isotropic-nematic $(I-N)$ and a nematic-columnar $(\mathrm{N}-\mathrm{C})$ phase transition [24], as predicted for sufficiently anisometric, monodisperse hard disks by computer simulations [25]. We address the influence of depletion attraction on both transitions, focusing on the effect on the transition densities, particle size fractionation, the appearance of attractioninduced phases, and multiphase coexistence.

\section{EXPERIMENT}

\section{A. Synthesis and characterization}

The suspension of platelets used in this paper consists of sterically stabilized gibbsite $\left[\mathrm{AL}(\mathrm{OH})_{3}\right]$ platelets dispersed in toluene. Following procedures described in Refs. [26-29], an aqueous suspension of gibbsite platelets is prepared by hydrothermal treatment of a $0.09 \mathrm{M} \mathrm{HCl}$ solution containing both $0.08 \mathrm{M}$ aluminum-sec-butoxide and $0.08 \mathrm{M}$ aluminumiso-propoxide. The platelets are subsequently grafted with a $M_{w} \approx 1000 \mathrm{~g} / \mathrm{mol}$ modified polyisobutylene (Shell code SAP230) and dispersed in toluene $[28,30]$, to provide the particles with a hard-core (short-range repulsive) interaction potential. The thickness of this polymer layer has been estimated as $4 \mathrm{~nm}$ [31].

Transmission electron microscopy (TEM) images were made using a Philips CM 10 electron microscope. From TEM micrographs (Fig. 1), the number-average diameter of the grafted platelets $\langle d\rangle$ was determined as the diameter of a circle with an area equal to that of the measured gibbsite core, plus twice the thickness of the polymer layer. We find $\langle d\rangle=208 \mathrm{~nm}$, with a standard deviation $\sigma=\sqrt{\left\langle d^{2}\right\rangle-\langle d\rangle^{2}}$ / $\langle d\rangle$ of $25 \%$. The determination of the thickness of the platelets by TEM is more complicated since the orientation of the platelets on the TEM grid is virtually always flat with respect to the grid surface. Therefore, some sample is flocculated deliberately by the addition of water in order to facilitate edgewise imaging of the platelets by TEM. The subsequent number-average thickness $\langle l\rangle$ of the grafted platelets is about $14 \mathrm{~nm}$, as obtained by adding the polymer layer thickness to the average TEM thickness of $6 \mathrm{~nm}$, which has a standard deviation of about $20 \%$. Hence the aspect ratio $\langle d\rangle /\langle l\rangle$ of the grafted particles is around 15 .

The nonadsorbing polymer is a trimethylsiloxyterminated polydimethylsiloxane with a weight average molar mass $M_{w}=4.2 \times 10^{5} \mathrm{~g} / \mathrm{mol}$. By extrapolation according to $R_{g} \propto M_{w}^{3 / 5}$, the radius of gyration $R_{g}$ of this polymer is estimated from Ref. [16] as roughly $33 \mathrm{~nm}$. This yields an effective diameter $\sigma_{\text {pol }}$ of the polymer coil of $74 \mathrm{~nm}$ [32]. The range of the depletion attraction is characterized by the ratio of the polymer coil diameter over the plate diameter $\sigma_{\text {pol }} / d_{\text {plate }} \approx 0.35$.

\section{B. Phase-separation experiments}

To explore the colloid-polymer phase diagram, samples of various plate-polymer concentrations are prepared by mixing weighed amounts of stock dispersion and stock polymer solution in $2 \mathrm{~mm}$ cuvettes. Subsequent variations in concentration are achieved either by dilution or by a combination of sedimentation and removal of the supernatant polymer solution. In the latter case, the polymer concentration is assumed to remain homogeneous throughout the sample after the sedimentation of the colloidal particles. Mixtures are thoroughly homogenized and left in a thermostated room in order to reach phase equilibrium.

The colloid volume fraction $\phi$, which includes the solvent present in the grafted polymer layer, is calculated from the mass concentration $c$ (determined by drying a known amount of dispersion at $75^{\circ} \mathrm{C}$ to constant weight) according to $[33,34]$

$$
\phi=\phi_{\text {core }} \frac{V_{p}}{V_{\text {core }}}=\frac{c(1-x)}{\rho_{\text {core }}} \frac{(d+2 \delta)^{2}(l+2 \delta)}{d^{2} l}=\frac{c}{\rho_{\mathrm{eff}}},
$$

where $V_{p}, V_{\text {core }}$ refers to the volume of the grafted particle and the gibbsite core, respectively. Further, $x$ is the polymer mass fraction of grafted particles as obtained from elemental analysis $(x=0.26), \rho_{\text {core }}$ the mass density of gibbsite [35], and $d, l$, and $\delta$ the diameter and thickness of the core and the polymer layer thickness, respectively. This yields the effective-mass density of the grafted particles $\rho_{\text {eff }}$ $=1.3 \mathrm{~g} / \mathrm{cm}^{3}$, with an estimated error of $10 \%$ due to the uncertainty in polymer layer thickness.

\section{SAXS experiments}

Samples exhibiting a columnar phase are investigated by small angle $\mathrm{x}$-ray scattering (SAXS, performed on the DUBBLE beam line at the ESRF, Grenoble, France. The $\mathrm{X}$-ray beam is highly monochromatic $\left(\Delta \lambda / \lambda \approx 2 \times 10^{-4}\right)$ and has a narrow focus (about $0.5 \times 0.5 \mathrm{~mm}^{2}$ ). The wavelength was fixed at $0.138 \mathrm{~nm}$. A $512 \times 512$ pixel detector was placed at about $8.5 \mathrm{~m}$ from the sample, yielding an accessible $q$ range of about $0.02-0.7 \mathrm{~nm}^{-1}$. The $q$ axis is calibrated using an oriented hydrated collagen fiber of rat tail tendon. The raw two-dimensional diffraction patterns are radially averaged after correction for transmission and background radiation. After preparation, samples for SAXS experiments are left in a thermostated room for 2 weeks in order to reach phase equilibrium. 


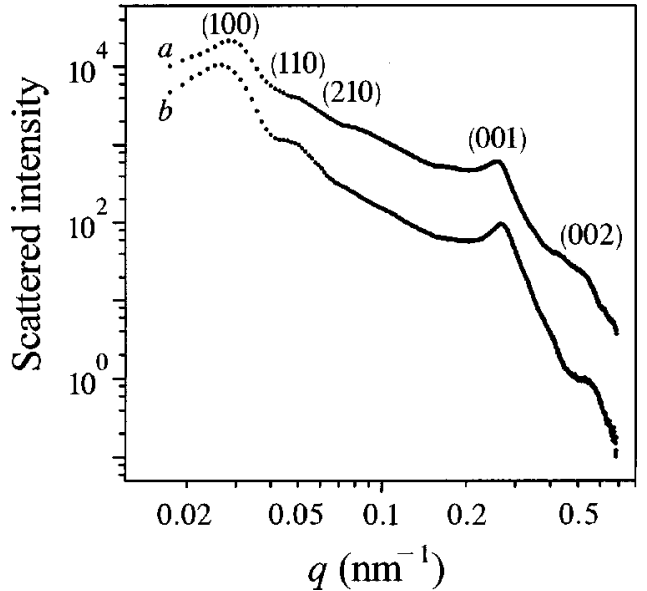

FIG. 2. SAXS patterns of a columnar phase in the case of (a) no nonadsorbing polymer added and (b) $0.9 \mathrm{~g} / \mathrm{l}$ polymer, at $\phi=0.45$ and 0.44 , respectively. Scattered intensities are in arbitrary units and curves are shifted vertically for clarity.

\section{RESULTS}

\section{A. Phase behavior without added polymer}

Upon increasing the platelet volume fraction, we observe an isotropic phase $(I)$, isotropic-nematic coexistence, a nematic phase $(N)$, nematic-columnar coexistence, and a columnar phase $(C)$. The isotropic-nematic $(I-N)$ coexistence region is located between $\phi=0.18$ and $\phi=0.21$. In order to compare these transition densities to results from computer simulations, we convert them to dimensionless densities of the form $n\left\langle d^{3}\right\rangle \approx(4 / \pi) \phi\left(1+2 \sigma^{2}\right)\langle d\rangle /\langle l\rangle$, with $n$ the number density of platelets (see also Ref. [28]). The density at the isotropic $\left(n_{I}\left\langle d_{I}^{3}\right\rangle\right)$ and the nematic $\left(n_{N}\left\langle d_{N}^{3}\right\rangle\right)$ boundary of the biphasic gap can be calculated using the suspension average $\langle d\rangle$ and $\langle l\rangle$ since, at these boundaries, the system has just turned monophasic such that size fractionation does not play a role. We obtain $\left(n_{I}\left\langle d_{I}^{3}\right\rangle, n_{N}\left\langle d_{N}^{3}\right\rangle\right)=(3.9,4.6)$. A number of computer simulations have addressed the $I-N$ transition densities for systems of hard platelike particles, using varying techniques and particle models [25,36-38]. The particle models that come closest to our experimental system were adopted in Gibbs ensemble Monte Carlo simulations by Bates and Frenkel, who considered polydisperse disks [38] as well as monodisperse hexagons [37]. By combining the results of these two studies, Bates [37] estimates that $\left.\left(n_{I}\right)\left\langle d_{I}^{3}\right\rangle, n_{N}\left\langle d_{N}^{3}\right\rangle\right)=(3.1,4.4)$ in the case of hexagons with $25 \%$ polydispersity, which is fairly close to the experimental transition densities. Such agreement supports the presumed hard-plate character of the experimental system.

The nematic-columnar $(\mathrm{N}-\mathrm{C})$ coexistence region is situated between $\phi=0.40$ and $\phi=0.48$. The SAXS pattern of the columnar phase, depicted by curve $a$ in Fig. 2, shows a number of scattering peaks. In the small $q$ regime, where the spacing $d=2 \pi / q$ is of the order of the diameter of the plates, we can distinguish one major peak and one or perhaps two additional peaks. The $q$ values of these peaks (Table I), whose $q$ ratio is like $1: \sqrt{3}: \sqrt{7}$, reveals that ordering in the plane of the plate diameters is hexagonal with the peaks corresponding to the (100), (110), and (210) reflections. Apparently the (200) reflection is relatively weak compared to its
TABLE I. $q$ values of the peak maxima as they appear in the SAXS patterns of the columnar phase with and without nonadsorbing polymer.

\begin{tabular}{lcccccc}
\hline \hline & & \multicolumn{5}{c}{$q\left(10^{-2} \mathrm{~nm}^{-1}\right)$} \\
\cline { 3 - 7 } & $c_{\text {pol }}(\mathrm{g} / \mathrm{l})$ & $(100)$ & $(110)$ & $(210)$ & $(001)$ & $(002)$ \\
\hline$a$ & 0 & 2.89 & 5.0 & 7.4 & 25.6 & \\
$b$ & 0.9 & 2.63 & 4.6 & 6.9 & 26.7 & 53 \\
\hline \hline
\end{tabular}

neighboring (110) and (210) peaks, such that it is not resolved in the scattering profile. From the $q$ values of the (100), (110), and (210) peaks we obtain the typical distance between the centers of the columns as $251( \pm 4) \mathrm{nm}$. The two peaks at much larger $q$ correspond to 1 and 0.5 times a spacing of roughly the plate thickness. We therefore identify them as the (001) and (002) reflections of the liquidlike order between the plates along the $z$ axis of a column of plates. The columnar signature of our $C$ phase, as opposed to a hexagonally ordered layerlike structure that would give rise to similar peaks, was demonstrated by unaffected $q$ values of the scattering peaks [24] upon tilting samples with respect to the x-ray beam $[39,40]$.

\section{B. Phase behavior with added polymer}

The addition of nonadsorbing polymer affects the phase diagram of the suspension of plates significantly. A striking effect is the distinct broadening of the isotropic-nematic gap (see Fig. 3), which means that the densities of the coexisting isotropic and nematic phases diverge strongly upon increasing the strength of the attraction. Purely nematic samples can actually be brought to isotropic-nematic coexistence by the addition of polymer.

Also, the depletion attraction introduces the distinction between a dilute isotropic $\left(I_{1}\right)$ and a more concentrated isotropic $\left(I_{2}\right)$ phase, replacing the isotropic phase $(I)$ that is observed in the absence of depletion attraction. We denote these phases by $I_{1}$ and $I_{2}$ to express their order in colloid

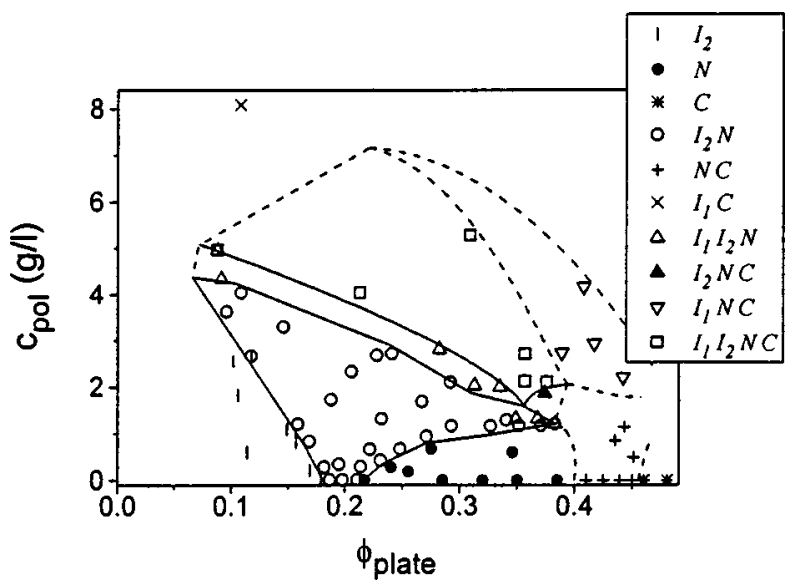

FIG. 3. The experimental phase diagram of the plate/polymer mixtures. Phase boundaries are indicated by lines, their shape and positioning being based on the data points they enclose, and on the consistency with surrounding phase regions. Lines are dashed in cases where the location of the phase boundary is not known precisely due to local scarcity of data points. 
volume fraction. The stability of two isotropic phases is analogous to the gas and liquid phase exhibited by suspensions of hard spheres and polymer, which occurs for polymer-sphere size ratios larger than about 0.3 [7]. In a recent computer simulation of infinitely thin platelets and interpenetrable polymer [41], the polymer-plate size ratio required for $I_{1}-I_{2}$ coexistence was found as the remarkably similar value of $\sigma_{\text {pol }} / d_{\text {plate }} \gtrsim 0.3$. In our experiment where $\sigma_{\text {pol }} / d_{\text {plate }} \approx 0.35$, the $I_{1}$ and $I_{2}$ phases are observed indeed, albeit in coexistence with the $N$ and/or $C$ phase.

As put forward in the introductory section, platelets are particularly sensitive to a depletion attraction since the overlap volume of depletion zones for parallel platelets is large compared to the case of spheres or rods. This qualitatively explains the low-polymer concentrations for which the phase behavior of the platelets is found to be affected by depletion attraction. The general expression for the depletion potential [Eq. (1)] has not yet been elaborated for the case of plateshaped colloids. For the sake of convenience, we estimate the maximum depth of the depletion potential here, that is, for parallel plates at contact. In this configuration the overlap volume of the depletion zones of the two platelets is approximated by $(\pi / 4) d_{\text {plate }}^{2} \sigma_{\text {pol }}$, where $d_{\text {plate }}$ is the diameter of the plate and $\sigma_{\text {pol }}$ that of the polymer. Applying Eq. (1) then yields the maximum depth of the depletion potential, to lowest order in the polymer density,

$$
W_{\mathrm{dep}}^{\max } \approx-k_{B} T \frac{n_{\mathrm{pol}}}{V} \frac{\pi}{4} \sigma_{\mathrm{pol}} d_{\mathrm{plate}}^{2}=-\frac{3}{2} k_{B} T \phi_{\mathrm{pol}}\left(\frac{d_{\mathrm{plate}}}{\sigma_{\mathrm{pol}}}\right)^{2},
$$

where $k_{B} T$ represents the thermal energy, $n_{\text {pol }} / V$ the number density of the polymer, and $\phi_{\mathrm{pol}}$ the volume fraction of the polymer. For hard spheres, the most obvious experimental criterion for the onset of depletion-induced phase transitions is the onset of gas-liquid coexistence, which typically requires a depth of the depletion potential of $-3 k_{B} T$. Likewise, for a plate-polymer mixture we may consider the lowest-polymer concentration for which the $I_{1}$ phase appears in the phase diagram. This is the case for $c_{\text {pol }} \approx 2 \mathrm{~g} / \mathrm{l}$. For the polymer size in this study, this corresponds to $\phi_{\text {pol }} \approx 0.6$ [using $\left.\phi_{\mathrm{pol}}=\left(c_{\mathrm{pol}} / M_{w}\right) \mathcal{N}_{A}(\pi / 6) \sigma_{\mathrm{pol}}^{3}\right]$ and hence $W^{\max } \approx$ $-7 k_{B} T$. For comparison we note that a similar strength of depletion attraction in the analogous case of spheres [2] would require an almost three times higher polymer concentration. Hence, the rather low-polymer concentrations required for depletion-induced phase transitions, and also the calculation in Eq. (3), demonstrate that platelets are rather sensitive to depletion attraction. Yet, by subtle tuning of the polymer concentration we are able to explore the platepolymer phase diagram without the interference of nonequilibrium states that may arise for more unbridled depletion attractions.

Compared to the pronounced broadening of the $I-N$ biphasic gap, the $\mathrm{N}-\mathrm{C}$ transition seems to be relatively less affected by depletion attraction. The boundary at the columnar side of the $\mathrm{N}-\mathrm{C}$ coexistence region remains largely undetermined however, as the increasing viscosity and time required for macroscopic phase separation limits the number of data points in the high-volume fraction/higher-polymer concentration regime. The structure of the columnar phase with and

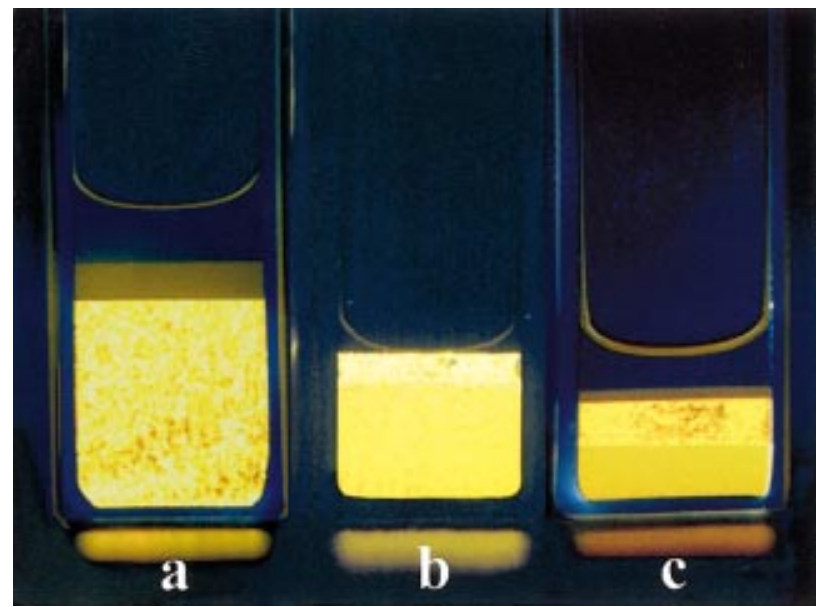

FIG. 4. (Color) Phase separated plate-polymer mixtures as observed between crossed polarizers. Depicted are (a) triple coexistence of the dilute isotropic $I_{1}$ (top), the more concentrated isotropic $I_{2}$ (middle), and a nematic phase $N$ (bottom) at the overall composition of $\phi_{\text {plate }}, c_{\text {pol }}=\{0.31,2.0\}$, (b) $I_{1}+N+C$ coexistence at $\{0.44,2.2\}$, and (c) $I_{1}+I_{2}+N+C$ coexistence at $\{0.31,5.3\}$.

without depletion attraction can be compared by the SAXS patterns shown in Fig. 2. Depletion attraction shifts the low $q$ peaks to slightly smaller $q$ values, i.e., the typical spacing between the columns increases. The peaks in the higher $q$ regime on the other hand, reflecting the liquidlike order between the platelets along the $z$ axis of the column, are shifted towards somewhat larger $q$. As both curves apply to a columnar phase at $\mathrm{N}-\mathrm{C}$ coexistence, the slight change in structure may be explained by depletion-enhanced fractionation, analogous to that at the $I-N$ transition. The shifts in $q$ values then suggest that, especially in the presence of depletion attraction, the columnar phase contains platelets of slightly larger diameter and smaller thickness than the coexisting nematic phase.

The overall topology of the plate-polymer phase diagram is characterized by a wealth of one-, two-, and three-phase equilibria and even a four-phase equilibrium (Fig. 4), each of these phase regions being based on possible combinations of the $I_{1}, I_{2}, N$, and $C$ phases. The origin of this notably rich

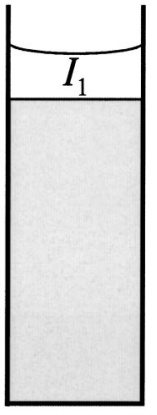

(I)

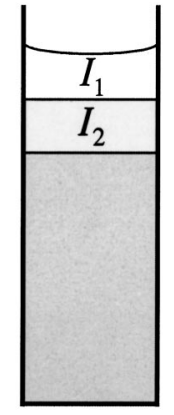

(II)

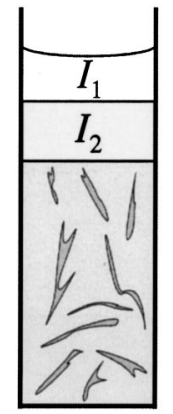

(III)

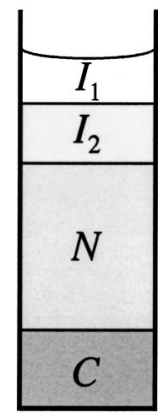

(IV)
FIG. 5. Schematic representation of the evolution of phase separation as typically observed in the four-phase coexistence region. For a sample with an overall composition of $\phi_{\text {plate }}, c_{\text {pol }}$ $=\{0.31,5.3\}$, the stages I-IV correspond to roughly $1 \mathrm{~h}$, a few hours, 1-2 days, and 2 weeks, respectively. 


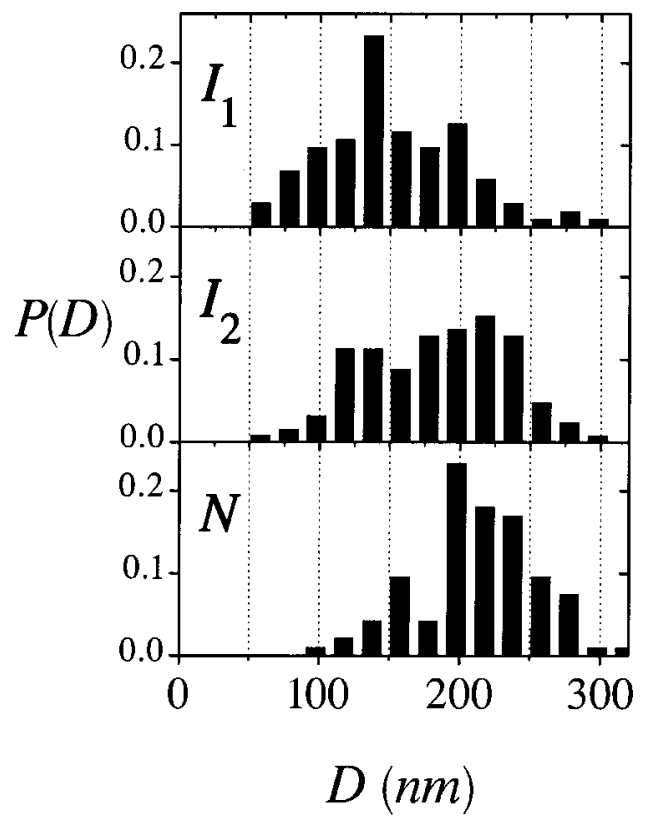

FIG. 6. The diameter distributions $P(D)$ in the coexisting phases of a $I_{1}+I_{2}+N$ equilibrium, as determined by TEM analysis. The distributions are based on around 100 particles each, and normalized such that $\sum P\left(D_{i}\right)=1$.

phase behavior is discussed in the next section. In view of a recent interest in the mechanism by which macroscopic phase separation evolves in sphere-polymer mixtures at triple coexistence [42], we include our observations for a plate-polymer mixture at four-phase coexistence in Fig. 5. The consecutive formation of the $I_{1}, I_{2}, N$, and $C$ phases involves essentially four stages. In stage I $(1 \mathrm{~h})$, the dilute $I_{1}$ phase separates from the turbid sample volume. In the next few hours (stage II) the turbid volume shrinks further, while leaving the $I_{2}$ phase behind. In stage III (after one to 2 days) turbid clouds are being formed in what develops as a nematic phase. The clouds subsequently sediment to the bottom in approximately 2 weeks, thus forming the columnar phase (stage IV). The way each of these phases is formed in the four-phase coexistence region is also largely representative for the way they are manifested in the corresponding threeand two-phase regions.

Along with the increasing density difference between the coexistent isotropic and nematic phase upon increasing the strength of the depletion attraction, TEM micrographs demonstrate that particle size fractionation between coexisting phases is also strongly enhanced compared to the polymerfree case. The diameter distribution of the coexistent phases in, for instance, a three-phase $\left(I_{1}+I_{2}+N\right)$ sample with a composition of about 0.05: 0.10: 0.85 by volume is depicted in Fig. 6. The number-average diameter in these three phases is 152,182 , and $214 \mathrm{~nm}$, respectively, with a subsequent relative standard deviation of $32 \%, 29 \%$, and $22 \%$. A sequence of removing isotropic phases followed by diluting the nematic with polymer solution, such that it just re-enters the $\left(I_{1}+I_{2}+N\right)$ region again, yields a suspension with a polydispersity in diameter of $17 \%$ after three cycles. As fractionation is almost absent in the absence of nonadsorbing polymer $[28,41]$, depletion-enhanced fractionation provides a convenient experimental tool to reduce the polydispersity of suspension.

\section{DISCUSSION}

The richness of the plate-polymer phase diagram raises the question how to explain the observed topology, including the four-phase region and its surrounding by three-, two- , and single phase regions. At first sight, such four-phase coexistence seems to conflict with the phase rule of Gibbs, which (at a given temperature) limits the maximum number of coexisting phases to three for a system of two components (plate + polymer). The observed four-phase coexistence actually demonstrates that the suspension of platelets, which comprises an almost infinite number of particles differing in diameter and thickness, cannot be described as a single species. The phase behavior of these polydisperse platelets can nevertheless be understood in terms of a bidisperse system, which consists of platelets of low- and high-aspect ratio, respectively. The in reality multidimensional plate-polymer phase diagram is then projected onto a three-dimensional phase space, set up by a $z$ axis for the polymer concentration and an $x$ and $y$ axis for the concentrations of the two species of plates. The $(x, z)$ and $(y, z)$ planes, each representing the phase diagram of monodisperse plates and polymer, are depicted schematically in Figs. 7(a) and 7(b). The global topology of these phase diagrams is inspired by theory and simulations of rodlike particles and polymer for different size

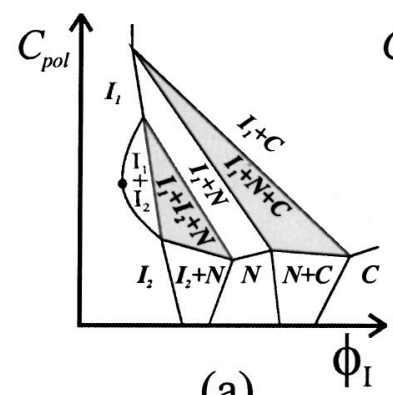

(a)

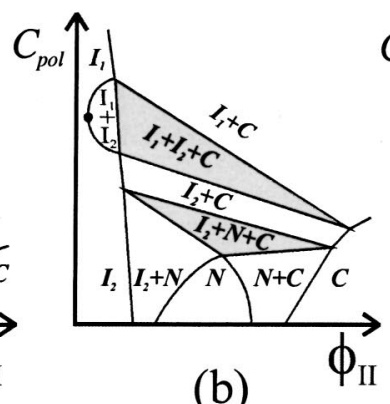

(b)

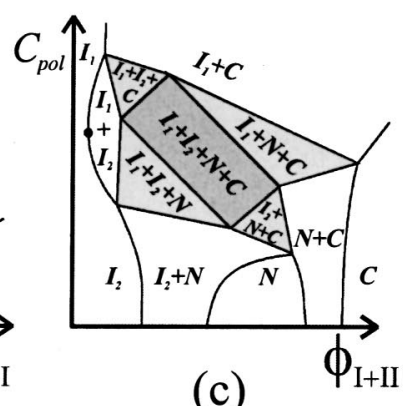

(c)

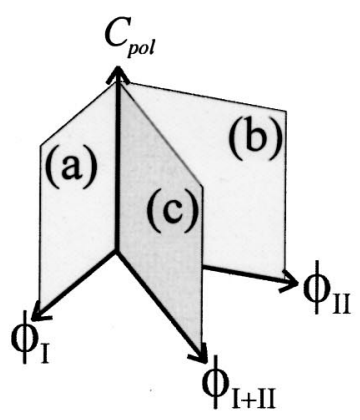

FIG. 7. Schematic perception of the plate-polymer phase diagram in case of (a) monodisperse platelets of lower-aspect ratio (species I), (b) monodisperse platelets of higher-aspect ratio (species II), and (c) a mixture of these two types of platelets. The relative position of each plane is sketched in the figure on the right. The $I_{1}-I_{2}$ coexistence regions on planes a and $\mathrm{b}$ are sketched not to extend up until the polymer axis but to end in a critical point (indicated by a closed circle). This is an arbitrary choice, although led by theoretical predictions for the rod-polymer phase diagram, which does not affect the main features of the proposed three-dimensional phase diagram. 
ratios $[19,20]$. The four different three-phasic regions that appear in these two planes are the projections of those threephase coexistence volumes that run through the threedimensional phase diagram. In the phase volume where all four three-phase volumes intersect, a tetrahedron-shaped four-phase $I_{1}-I_{2}-N-C$ region appears. Our experimental phase diagram on the other hand is set up by a $z$ axis for the polymer concentration, and another axis in the $x, y$ plane for the concentration of the (virtually) bidisperse mixture of the two species of plates. The projection of the four-, three-, two- and monophasic regions onto this experimental plane is sketched in Fig. 7(c), showing qualitatively the same features as the experimentally observed phase diagram. The threedimensional representation of the plate-polymer phase diagram also visualizes why $I_{1}-N$ and $I_{2}-C$ coexistence is not observed in the experiment. This is because these phase regions, although present in the monodisperse $x, z$ and $y, z$ planes, are squeezed out between three-phasic volumes outside these monodisperse planes and therefore do not intersect with the experimental plane.

\section{CONCLUSIONS}

We have studied the influence of nonadsorbing polymer on the phase behavior of a suspension of plates. Major effects are the pronounced broadening of the isotropic-nematic gap and the appearance of an additional isotropic phase $I_{1}$. Furthermore, particle size fractionation is strongly enhanced compared to the polymer-free case, which provides a convenient experimental tool to reduce the polydispersity of the suspensions. The overall topology of the plate-polymer phase diagram includes a large variety of phase regions where the $I_{1}, I_{2}, N$, and $C$ phases coexist in different combinations. We show that the richness of the plate-polymer phase diagram can be understood by a virtual projection of the polydisperse suspension of plates onto a bidisperse mixture of plates of different aspect ratio. Further insight in the phase behavior of plate-polymer mixtures demands study by computer simulation, addressing the role of attraction and polydispersity on the stability of the nematic, columnar, and smectic liquid-crystal phases.

\section{ACKNOWLEDGMENTS}

The authors would like to thank K. Kassapidou for assistance with the SAXS experiments, I. Dolbnya and W. Bras for technical support at DUBBLE, and M. Bates for discussions on his recent simulations of plate-polymer mixtures. Our work was supported by the Foundation for Fundamental Research on Matter (FOM) and the Netherlands Organization for the Advancement of Research (NWO).
[1] S. Asakura and F. Oosawa, J. Chem. Phys. 22, 1255 (1954).

[2] A. Vrij, Pure Appl. Chem. 48, 471 (1976).

[3] C. F. Vester, Kolloid-Z. 84, 63 (1938).

[4] R. Fahreus, Acta Med. Scand., Suppl. 55, 1 (1921).

[5] C. Cowell, R. Lin-In-On, and B. Vincent, J. Chem. Soc., Faraday Trans. 1 74, 337 (1978).

[6] H. N. W. de Hek and A. Vrij, J. Colloid Interface Sci. 70, 592 (1979)

[7] H. Lekkerkerker et al., Europhys. Lett. 20, 559 (1992).

[8] A. Gast, C. Hall, and W. Russel, J. Colloid Interface Sci. 96, 251 (1983)

[9] E. Meijer and D. Frenkel, J. Chem. Phys. 100, 6873 (1994).

[10] P. Bolhuis and D. Frenkel, J. Chem. Phys. 101, 9869 (1994).

[11] W. Poon, Curr. Opin. Colloid Interface Sci. 3, 593 (1998).

[12] S. M. Illet, A. Orrock, W. C. K. Poon, and P. N. Pusey, Phys. Rev. E 51, 1344 (1995).

[13] M. A. Faers and P. F. Luckham, Langmuir 15, 2922 (1997).

[14] L. Onsager, Ann. N.Y. Acad. Sci. 51, 627 (1949).

[15] S. S. Cohen, J. Biol. Chem. 144, 353 (1942).

[16] J. Buitenhuis et al., J. Colloid Interface Sci. 175, 46 (1995).

[17] M. Adams and S. Fraden, Biophys. J. 74, 669 (1998).

[18] M. Adams, Z. Dogic, S. L. Keller, and S. Fraden, Nature (London) 393, 349 (1998).

[19] H. Lekkerkerker and A. Stroobants, Nuovo Cimento D 16, 949 (1994).

[20] P. G. Bolhuis, A. Stroobants, D. Frenkel, and H. N. W. Lekkerkerker, J. Chem. Phys. 5, 1551 (1997).

[21] M. Morvan et al., Langmuir 10, 2566 (1994).

[22] A. Mourchid et al., Langmuir 11, 1942 (1995).

[23] J. C. P. Gabriel, C. Sanchez, and P. Davidson, J. Phys. Chem. 100, 11139 (1996)
[24] F. M. van der Kooij, K. Kassapidou, and H. N. W. Lekkerkerker, Nature (London) 406, 868 (2000).

[25] J. A. C. Veerman and D. Frenkel, Phys. Rev. A 45, 5632 (1992).

[26] A. P. Philipse, A. Nechifor, and C. Patmamanoharan, Langmuir 10, 4451 (1994).

[27] A. Wierenga, T. Lenstra, and A. P. Philipse, Colloids Surface 134, 359 (1998).

[28] F. M. van der Kooij and H. N. W. Lekkerkerker, J. Phys. Chem. 102, 7829 (1998).

[29] F. M. van der Kooij and H. N. W. Lekkerkerker, Phys. Rev. Lett. 84, 781 (2000).

[30] P. A. Buining, Y. S. J. Veldhuizen, C. Pathmamanoharan, and H. W. W. Lekkerkerker, Colloids Surface 64, 47 (1992).

[31] C. Smits, W. J. Briels, J. K. G. Dhont, and H. N. W. Lekkerkerker, Prog. Colloid Polym. Sci. 79, 287 (1989).

[32] H. de Hek and A. Vrij, J. Colloid Interface Sci. 84, 409 (1981).

[33] F. van der Kooij, E. Boek, and A. Philipse (unpublished).

[34] M. P. B. van Bruggen, J. K. G. Dhont, and H. N. W. Lekkerkerker, Macromolecules 32, 2256 (1999).

[35] W. H. Gitzen, Alumina as a Ceramic Material (The American Ceramic Society, Columbus, 1970).

[36] R. Eppenga and D. Frenkel, Mol. Phys. 52, 1303 (1984).

[37] M. A. Bates, J. Chem. Phys. 111, 1732 (1999).

[38] M. A. Bates and D. Frenkel, J. Chem. Phys. 110, 6553 (1999).

[39] A. Guinier, X-ray Diffraction in Crystals, Imperfect Crystals, and Amorphous Bodies (Dover, New York, 1994), p. 169.

[40] A. B. D. Brown, C. Ferrero, T. Narayanan, and A. Rennie, Eur. Phys. J. B 11, 481 (1999).

[41] M. A. Bates and D. Frenkel, Phys. Rev. E (to be published).

[42] W. C. K. Poon et al., Phys. Rev. Lett. 83, 1239 (1999). 